\title{
El embarazo precoz: no querido pero deseado
}

\section{Early pregnancy: not wanted but wished for}

Miriam E. Trujillo Mariño*

En los últimos años la tasa de fecundidad global entre las mujeres adultas ha descendido notablemente, en tanto entre las adolescentes se ha incrementado, al decir de Matilde Maddaleno (1995) en un artículo para la Organización Panamericana de la Salud; de igual manera opina Marcelo Dávalos (2006) al manifestar que en el país ha descendido de 6,76 a 3,61 en el último medio siglo anterior, en tanto que la "tasa de fecundidad especifica" de adolescentes en América ha aumentado. De cuatro embarazos reportados uno es adolescente, por lo que podríamos colegir que de cien embarazos, veinticinco son precoces.

Frente a esto, reportes del Ministerio de Salud Pública (2006), mencionan que parte de ello, se debe a que la edad de inicio de la relación sexual en las mujeres ha disminuido a los catorce años, y en el hombre de quince años; $y$ como consecuencia inmediata tenemos que ocho de cada diez embarazos adolescentes no son deseados, ocho de cada diez adolescentes no reinician sus estudios después del parto, el 100\% de madres adolescentes viven con sus padres o suegros, el 95\% de madres jóvenes no tienen trabajo durante el periodo de gestación, tres de cada diez tienen un segundo hijo antes de terminar la adolescencia, lo cual revisaremos más adelante.

Reflexionar el "embarazo precoz" como problema social comienza por el análisis de la información y datos estadísticos (Sánchez Parga, 2004), así lo denuncia la realidad y la opinión pública como urgencia social “(...) en Ecuador existe el $30 \%$ de riesgo de muerte en las madres adolescentes porque no reciben información ni educación que respalde a las jóvenes y les ofrezcan orientación médica y psicológica" (2006) además de situaciones vinculadas como: hijos no deseados y abortos; abandono o adopción de hijos de madres adolescentes; madres solteras; matrimonios forzados; deserción escolar, entre otros.

La salud reproductiva de este grupo poblacional "es uno de los principales problemas de políticas sociales en salud pública en el país, considerando que el

\footnotetext{
* Resumen de una investigación presentada para la tesis de postgrado. UPS. Quito. Maestría en Políticas Públicas de Infancia y Adolescencia. Enfermera en la Maternidad Isidro Ayora. Quito. e-mail: myriamelisabethtru@hotmail.com
} 
embarazo precoz provoca afectaciones de la salud física, psíquica y social de la madre, en su pareja, en el niño e involucra a la estructura familiar y social" (Naranjo, 2000). A esto se suman factores como:

- Deterioro de las condiciones de vida de la población -efecto de la profundización de la crisis económica, política, social y ética-.

- Cambios en el funcionamiento familiar -pobreza, desempleo, fenómenos de aculturación, problemática educativa, malnutrición-.

- Desinformación y la ignorancia.

Entre otros, pueden ser influyentes en el comportamiento de las adolescentes y por ende incidir en el incremento de embarazos precoces. En este sentido se puede afirmar que el grupo de mayor riesgo social, en la actualidad es el grupo poblacional de adolescentes, quienes no sólo no han sido considerados entre las prioridades para el diseño de políticas públicas de desarrollo, sino que aparecen socialmente con una estela de estigmas y temores, "ausencias de derechos", y confusiones de cómo actuar y comprender sus problemáticas.

Frente a esta realidad el presente trabajo investigativo centra su esfuerzo en procurar una comprensión y explicación sociológica del embarazo precoz o adolescente, que permite acercarse a este factor de riesgo que afecta y limita su desarrollo personal, familiar y social. Como se verá, "es evidente que el embarazo precoz es un síntoma de una patología social, que puede y debe prevenirse si los estados y los gobiernos le dan la importancia y relevancia debida" (Naranjo, 2000). En sí, se busca identificar luces que permitan perfilar y definir políticas sociales focalizadas en sus familias y afecciones.

\section{Definición y delimitación del problema}

La maternidad en los años adolescentes se ha mantenido alta en las últimas dos décadas en el Ecuador, las estadísticas vitales en el 2002, último año con información, dan cuenta que 12.300 adolescentes de doce a diecisiete años tuvieron hijos; $y$, según la encuesta quinquenal de salud materna, la maternidad entre los quince y diecinueve años, aumentó su frecuencia en los últimos diez años en la Costa y la Amazonía más que en la Sierra, siendo las provincias de los Ríos, Esmeraldas, Manabí y en la Amazonía, Sucumbíos y Zamora Chin- 
chipe las que sobresalen con los más altos porcentajes de partos adolescentes. En tanto que en la Sierra, Azuay tiene el menor número porcentual (Naranjo, 2000). En 1999, una de cada seis jóvenes de quince a diecinueve años de edad -cerca de 90.000 jóvenes- había tenido por lo menos un hijo (2004).

No todos estos embarazos son deseados. Es más, la proporción de embarazos no previstos y no deseados entre los jóvenes de quince a diecinueve años creció en los últimos años: pasó del 21\% en 1994 al 28\% en 1999. Para el 2004, según reporta el Consejo Nacional de Salud en el "Documento Política Nacional de Salud y Derechos reproductivos", el 20\% de las adolescentes y jóvenes entre quince y veinticuatro años, han estado embarazadas por lo menos una vez, este documento también revela que de los 128.530 partos únicos espontáneos el $22 \%$ fueron de madres adolescentes, y de los 556 abortos espontáneos 96 (17\%) fueron en adolescentes y de los 18.381 abortos no especificados, 2.949 (16\%) fueron de adolescentes (Ministerio de Salud, 2005: 31). El embarazo precoz tiene, en relación al número de nacimientos, una frecuencia similar tanto en el área rural como en la urbana.

Por ello, desde 1988 el Hospital Gíneco Obstétrico "Isidro Ayora" crea una unidad de consulta externa diferenciada para adolescentes embarazadas, para 1994 es equipado y se convierte en el Primer "Centro piloto de Atención a la población adolescente" por parte del Ministerio de Salud Pública. Diez años después de su inicio, en 1998, inicia, con financiamiento español la "Unidad de Atención Integral para adolescentes" (HGOIA-AECI), reportando que en la década anterior "se atendió 153.621 mujeres, de las cuales, el 21\% (31.911) fueron adolescentes, fluctuando anualmente entre el 17\% y el 25\%" (Naranjo, 2000).

Es difícil determinar, con exactitud, las causas del embarazo precoz, puesto que estas son multicausales e interrelacionadas, su registro puede no ser exacto, como se manifestó en párrafos anteriores, en tanto pueden existir sub-registros especialmente en el área rural, en vista de ello se establecen los registros del proyecto HGOIA-AECI del Hospital Gíneco-Obstétrico de Quito para determinar las características y análisis del objeto de estudio, planteándose cuestionamientos generales y específicos que ayuden a enfocar la investigación.

¿Qué caracterizó el embarazo precoz en los reportes del proyecto HGOIAAECI en su primer año de funcionamiento 1999 y en el año 2005? Con este cuestionamiento general se pretendió demarcar y visualizar en conjunto la población 
adolescente de "embarazo precoz" en el marco de esta investigación. Sin embargo, para precisar el objeto de investigación, se formularon cuestionamientos más puntuales para esta situación en adolescentes atendidas por el proyecto mencionado, y posteriormente poder definir estrategias de políticas sociales de ayuda.

Para ello se plantearon las siguientes preguntas:

1. ¿Qué factores sociales y familiares son determinantes para la presencia de embarazo precoz entre adolescentes tratadas por el proyecto HGOIA-AECI en los años 1999 y 2005?

2. ¿Cuáles son los "riesgos adolescentes" personales y sociales vinculadas al embarazo precoz entre adolescentes tratadas por el proyecto HGOIAAECI.

Para responder estas preguntas se toma como base de la investigación al Hospital Gineceo Obstétrico "Isidro Ayora", y el proyecto "Atención Integral al Adolescente", símbolo de atención especializada a la adolescente embarazada.

\section{Objetivos e Hipótesis}

A partir de la concreción de las preguntas se procedió a la definición de objetivos, tomando como objetivo general: "analizar la caracterización del embarazo precoz entre adolescentes tratadas por el proyecto HGOIA-AECI en los años 1999 y 2005", y como objetivos específicos los siguientes:

1. Identificar y analizar los factores sociales y familiares determinantes para la presencia de embarazo precoz entre adolescentes tratadas por el proyecto HGOIA-AECI en los años 1999 y 2005.

2. Identificar y caracterizar los "riesgos adolescentes" personales y sociales vinculadas al embarazo precoz entre adolescentes tratadas por el proyecto HGOIA-AECI.

3. Indagar en qué medida el embarazo puede no ser querido, pero si profundamente deseado. Para ello se completó el perfil objetivo socioeconómico de la embarazada con el perfil subjetivo del embarazo; es decir cómo la joven ha vivido su embarazo.

Como hipótesis se plantea que: el embarazo precoz está determinado por factores sociales y familiares tales como el deterioro de las condiciones de vida 
de la población-efecto de la profundización de la crisis económica, política, social y ética-, los cambios en el funcionamiento familiar -producto de la exclusión, pobreza, desempleo, fenómenos de aculturación, problemática educativa, malnutrición-, y desinformación e ignorancia en sexualidad y prevención. La adolescente con embarazo precoz se caracteriza por estar vinculada a riesgos adolescentes personales y sociales como son: hijos no deseados y abortos, abandono o adopción de sus hijos en gestación, madres solteras, matrimonios forzados, deserción escolar, y desempleo.

Estos datos se obtuvieron de dos fuentes de información obtenidas en los meses de julio, agosto y septiembre del año 1999 y 2005 de los mismos meses, a saberse el Formulario No. 028.1 / 99 (CLAP. Anexo No 1) instrumento del Proyecto y el Ministerio de Salud Pública, y guía de entrevista a cincuenta adolescentes embarazadas y adolescentes que acuden a la consulta médica después del parto, para explorar datos conexos al formulario anterior citado (anexo $\mathrm{N}^{\mathrm{o}} 2$ ).

\section{Marco Teórico y Fundamentación}

Este subtítulo, presenta las ideas generales del proceso de investigación, la realidad del problema social a la problemática sociológica del embarazo precoz, procurando la interpretación a la luz de su tratamiento como un hecho sociológico que afecta el futuro de la sociedad, familia y la adolescente embarazada.

En el marco del análisis sociológico se utiliza el concepto general de Durkheim, quien concibe al "hecho social" como las:

Formas de actuar, pensar y sentir que son objetivas y externas al individuo, que tienen un poder de coacción, por el que se imponen al mismo individuo quiera o no quiera; son fruto de la acción múltiple y simultánea de individuos, son por tanto modelos colectivos preexistentes impuestos, elaborados por generaciones pretéritas. Son el sustrato social, de lo que Durkheim llama la conciencia colectiva, suelen formularse de manera oral o por escrito, como normas que se transmiten por la educación (aforismos, leyes morales, jurídicas); quedándose fijadas en obras objetivas (estilos de arte, culturas, modas, etcétera). Aunque, en parte modificables por nuevas acciones individuales, ofrecen resistencia al cambio. 
Difícilmente la sociedad se permite comprender y explicar los "hechos sociales" como una "cuestión social", en cuanto, así como la pobreza, el embarazo precoz en adolescentes le significa indudablemente cuestionarse como sociedad productora de aquel hecho. De ahí que:

(...) como los "embarazos precoces" tengan que ser pensados, comprendidos y explicados, no tanto desde las jóvenes adolescentes que los provocan, cuanto desde la sociedad que los propicia, los produce, los generaliza, los vuelve necesarios(?), ante otras alternativas posibles de integración adolescente. En cuanto productor de sociedad, el estado ha sido siempre el principal precursor de hechos sociales en la sociedad societal, y han sido sobre todo sus políticas y programas sociales, los que más han contribuido a la producción social tanto en la familia como más actualmente en la infancia (Sánchez Parga, 2004). (...) La cual es un hecho social y un objeto sociológico, esto significa comprender y explicar la infancia en todas sus formas y procesos, en cuanto hecha por un sistema de acciones y relaciones sociales, de las estructuras y procesos sociales, pero también en cuanto que la misma infancia hace sociedad, contribuye a interpretarla. (Sánchez Parga, 2004: 26-41).

\subsection{Sexualidad adolescente}

$\mathrm{Al}$ abreviarse, en la sociedad actual, el periodo de la infancia con una entrada más precoz en la fase adolescente, al niño se le reduce el tiempo de su maduración efectiva infantil, al mismo se le iniciará prematuramente en la vida y las relaciones sexuales, para las cuales no se encontraría afectivamente maduro (Sánchez Parga, 2004). Esto daría lugar a un desfase entre el desarrollo afectivo trunco o incompleto, respecto del modelo de sociedad anterior, y una precoz iniciación a la vida sexual. En tanto, que en sociedades más tradicionales el adolescente debía quedar preservado el mayor tiempo posible de la sexualidad y de su pleno ejercicio, el cual estaba reservados estrictamente a los adultos, debido precisamente a las responsabilidades que acarrea (condiciones materiales y personales para mantener una familia de manera autónoma).

La sociedad moderna no sólo hace de la sexualidad un derecho, prescindiendo de los deberes y obligaciones que tal derecho entraña, sino que ha liberado de tal manera la sexualidad y la ha hecho tan pública que las relaciones sexuales alcanzan niveles de edad cada vez más tempranas. El deseo de experi- 
mentar lo desconocido y el placer es propio de esta edad, pero sin información adecuada, ni profesional ni apoyo emocional, el inicio de la sexualidad expone a los y las adolescentes a enfermedades y al embarazo precoz, entonces ¿Dónde queda su derecho al futuro? ¿A avanzar en su desarrollo emocional e intelectual y definir sus proyectos futuros?

Cuanto mayor o más intensa es la exposición de los jóvenes a los mensajes de contenido sexual explícito, tiende a descender más la edad de la primera relación sexual. En el fondo se combinan una serie de elementos: la liberación y publicidad de la sexualidad sin recurrir a políticas represivas y censuradoras; ausencia de políticas y programas eficaces, no filantrópicos y moralizantes (que vayan más allá de convencionales educaciones sexuales, de la salud reproductiva y de la promoción de preservativos), y no simplemente limitados a prevenir el embarazo, sino a formar sexualmente al adolescente.

\subsection{Embarazo precoz}

En el marco del presente estudio se concibe el "embarazo precoz" como el embarazo que ocurre dentro de los dos años de edad ginecológica, entendiéndose por tal al tiempo transcurrido desde la "menarquía", y/o cuando la adolescente es aún dependiente de su núcleo familiar de origen, al decir de Ramiro Molina Cortés (Coll y Dulanto, 2001). Mas allá del concepto médico el embarazo precoz plantea dos problemáticas sociológicas: la femenina "el deseo de un embarazo no querido", en los cuales los adolescentes pretenderían encontrar una prueba de su sexualidad y capacidad puesta entredicho por la sociedad actual y por otra, la adolescente qué con signos visibles trasciende a madre cuando "deja de ser hija para hacerse madre" (Sánchez Parga, 2004).

Por otra parte, el "Consenso Social sobre Embarazo Precoz y Maternidad Adolescente" se ha modificado con el transcurso del tiempo y los cambios sociales que han tenido lugar. De este modo, desde hace tres décadas, este fenómeno se ha convertido en preocupación urgente para varios sectores de la sociedad básicamente urbana; desde el punto de vista de la salud por la mayor incidencia de resultados peri natal desfavorables, cuando la joven lleva adelante el embarazo, o por las implicaciones de aborto, si decide interrumpirlo. Desde la perspectiva psicosocial, el embarazo precoz preocupa por las consecuencias adversas que tiene en muchos casos sobre las adolescentes y sus familias. 
La percepción y preocupación por los embarazos precoces y su aumento, no tanto en número sino en cuanto problema, es debido al mayor abandono por parte de la sociedad a las adolescentes madres y sus hijos. Estas medidas no deben ocultar la problemática de fondo existente, una realidad convertida en "indicador de la desinserción social" tanto de los como de las adolescentes. Antes la madre adolescente y su hijo eran mejor integrados tanto en el ámbito de la propia familia como en la misma sociedad, mientras que en la actualidad son objeto no sólo de abandono sino incluso de rechazo. Fenómeno que habrá de ser interpretado en cuanto efecto de una generalizada exclusión social de la que son víctimas los grupos o sectores más frágiles de la misma sociedad.

La estadística demuestra, de manera general, que un $80 \%$ de los embarazos precoces pertenecen a adolescentes de sectores pobres, populares o marginales, lo cual indica que para dichas jóvenes la maternidad respondería a una estrategia de inserción social, de afirmar una identidad o un proyecto de vida. Aún cuando el embarazo precoz lejos de resolver el problema de la inserción social tiende más bien a agravarlo, "la adolescente busca en la maternidad una forma de seguridad y autonomía personal dentro de la propia familia y aún de la sociedad" (Sanchez Parga, 2004).

Son estas situaciones de orden sociológico las que permiten comprender e interpretar esa situación paradójica entre un "embarazo no deseado" y una "maternidad deseada", entre lo que no se quiere y lo que se desea. En este sentido resulta obvio que tras una estrategia afectiva y de integración social cifrada en el embarazo y la maternidad, la adolescente no desea las consecuencias de tal embarazo y maternidad, las cuales tienden a agravar su marginalización social.

El embarazo en la etapa de la adolescencia se considera como una situación problemática que origina conflictos para los sujetos involucrados, excepto cuando el tener un hijo forme parte del proyecto de vida de la pareja adolescente.

\section{Factores de riesgo del embarazo precoz}

Las estadísticas, indican que cuanto mayor es la integración de la mujer en la sociedad y las posibilidades-perspectivas de integración social de las jóvenes, más baja es la tasa de maternidad y de embarazos precoces. Por ello, 
la tasa de fecundidad adolescente es más alta en países en desarrollo y entre clases sociales menos favorecidas, haciendo pensar que se trata de un fenómeno transitorio, porque de mejorarse las condiciones, esta podría descender. Igualmente podríamos entrever factores de orden socioeconómico conectados con la relación entre adolescente, su integración social y su proyecto de devenir adulto. Para otros investigadores este fenómeno está muy lejos de ser una problemática transitoria, las tasas de fecundidad adolescente están cada vez más altas, y el deterioro de las condiciones socioeconómicas globales hace que se dude sobre la posibilidad de que la mayoría de los países realicen considerables inversiones en sus sistemas educativos y de salud, para alcanzar la cobertura que el problema demanda.

Según Jorge Naranjo (2000), el embarazo precoz, se produce por múltiples factores de riesgo identificados por los siguientes aspectos:

a. El contexto sociocultural y económico: migración; actitud social punitiva frente al embarazo, bajo nivel educativo, mayor dependencia económica, deserción escolar.

b. El contexto familiar: estilo de vida de riesgo, familia disfuncional, actitud familiar negativa frente al embarazo. A su decir, esta es de mucha importancia, muchas adolescentes se embarazan buscando salir e independizarse de su familia, más no lo consiguen y al contrario su situación familiar se agrava en cuanto se incrementa su dependencia.

c. El contexto individual: embarazo no deseado; falta de apoyo de la pareja, inmadurez y ausencia de proyecto de vida individual, incapacidad de conducta preventiva, pobre autoestima y de-privación afectiva, debilidad mental, estrés agudo crónico, otras conductas de riesgo que estuvieren asociadas.

En general las adolescentes que se embarazan en la adolescencia presentan una incidencia más elevada de trastornos emocionales; en este grupo es más común la negación del embarazo y de los trastornos emocionales. El foco de preocupación es el propio cuerpo y la separación de la madre, en muchas niñas menores de quince años el embarazo a menudo es la respuesta contra fóbica a la dependencia (nunca satisfecha) de la madre. Y lo es a manera de experimento o prueba con el propio cuerpo misterioso. 


\section{Perfil Socio Familiar de la Adolescente Embarazada}

En el capítulo precedente, se indicaron algunos criterios que motivaron la investigación presente, para plasmar, no obstante, el análisis de acuerdo a los elementos descritos es necesario precisar algunas pautas como las siguientes:

Para la elaboración de este trabajo, en la primera etapa de recolección de datos, se utilizó la historia clínica del CLAP/OPS con entrevista a 200 adolescentes que fueron atendidas en el periodo julio a septiembre de los años 1999 y 2005. Para el análisis y construcción del perfil socio familiar de la adolescente embarazada se ha utilizado la segmentación propia del instrumento aplicado, es decir:

1. Caracterización y procedencia: detalla información del origen, migración y situación actual de la adolescente.

2. Factores de riesgo personales y familiares: en el cual se detalla información relevante del entorno socio afectivo y médico en el que se desarrolla el embarazo de la adolescente.

3. Familia: nos devela el entorno y características genotípicas de la familia de la adolescente.

4. Vivienda, educación, trabajo, vida social y hábitos de la adolescente en relación a su desarrollo y socialización.

5. Sexualidad y situación psicoemocional: en los cuales se identifica un entorno de riesgo adolescente vinculados al embarazo precoz.

\section{Adolescentes atendidas en el proyecto HGOIA-AECI}

La unidad de atención integral para adolescentes, HGOIA-AECI, ha tenido un crecimiento muy importante desde su creación, como podemos observar en la tabla en el año 1999 de un total de 20.636 atenciones, 1.846 (8,95\%) fueron adolescentes que acudieron a recibir atención Prenatal; hasta octubre del año 2005, de 23.367 atenciones, $6.175(26,43 \%)$ son adolescentes que fueron por su control en la etapa gestante, la cobertura de atención se ha incrementado casi cuatro veces.

Como se ilustra, desde el año 1999 al 2005, hubo un incremento alarmante de atención de adolescentes en la unidad del proyecto HGOIA-AECI del Hospital Gíneco Obstétrico "Isidro Ayora", en relación a la población total, 17,5\%, lo que representa respecto a las adolescentes atendidas en 1999 y el 2005 de un $33,5 \%$, este incremento es entendible desde el punto de vista que el proyecto se inaugura en el año que se toma como partida para este análisis. 
En el cuadro siguiente, el ingreso de adolescentes para la atención en la unidad del Proyecto HGOIA-AECI, ha sido seccionado el periodo específico de análisis e investigación, lo cual permitió recolectar datos del periodo mensual comprendido entre julio y septiembre de los años mencionados. Con la ayuda del Departamento de Estadística y Admisión, se realizó comparación entre la tabla y el gráfico que muestra en el año 1999 un total de 6.102 adolescentes atendidas, $653(10,70 \%)$ acudieron a recibir atención Prenatal; y en el año 2005 de 8.445 atenciones, $2.350(27,83 \%)$ acudieron por control en la etapa gestante, es decir, se mantiene la tendencia general de incremento de cobertura.

\section{Crecimiento de la atención de adolescentes en la consulta externa, julio a septiembre año 1999 y 2005}

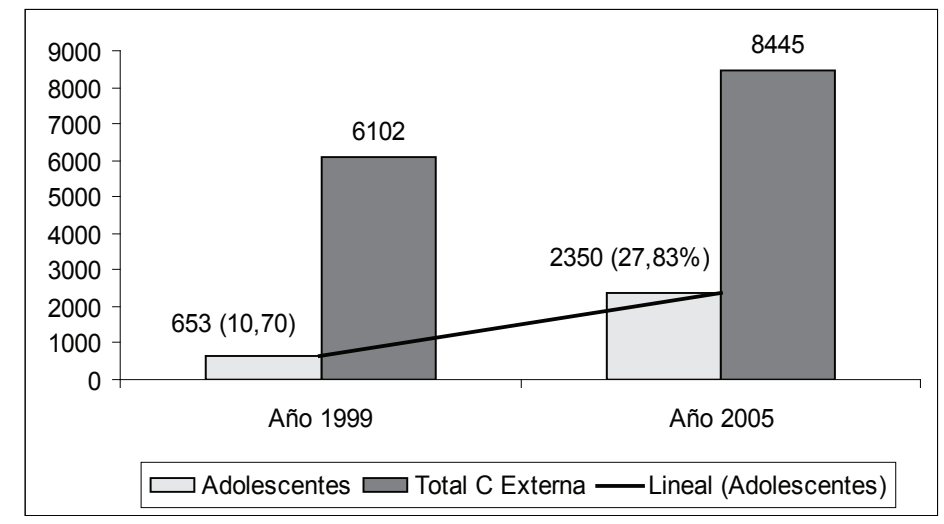

Elaborado por: Myriam Trujillo.

Fuente: Departamento de Estadística y Admisión del HGOIA. Datos Consulta Externa 1999 y 2005.

\section{Caracterización y Procedencia de la Adolescente}

Seleccionado y revisado doscientas fichas, elegidas entre los reportes de los meses de julio a septiembre entre los años objetos de estudio. Para una mejor comprensión de la "caracterización de la adolescente", el conjunto de análisis 
inicial se distribuyó en dos subgrupos de edades, según los parámetros definidos por la OPS-OMS, de diez a catorce años y quince a diecinueve años. Si bien, para el 2005 el conjunto de adolescentes del segundo grupo sigue siendo la población predominante en embarazo precoz, también es alarmante el incremento de casos en adolescentes menores, aunque numéricamente no es muy considerable, el incremento porcentual si lo es, pues asciende al 2,5\% en comparación al año 1999.

Esta segmentación en dos grupos de análisis, se hace necesaria para caracterizar a la población adolescente que durante los años de análisis solicitaron atención en la unidad de consulta externa del proyecto, y como se describe esta población es fundamentalmente la que en el ámbito medico-social se denomina adolescentes mayores. Para estimar en el estudio el vínculo de la adolescente ante su situación, se revisa en el análisis: quien le acompañó a su control de embarazo; con la finalidad de saber el nivel de apoyo y cercanía de su entorno. En 1999 el 18,5\% expresó que acudía sola a la consulta, a diferencia del año 2005 esta respuesta se incrementó en 11,5 puntos, es decir, $30 \%$ de las adolescentes respondió en los mismos términos.

¿Quién acompaño a la consulta?

\begin{tabular}{|l|c|c|c|c|}
\hline Acompañante & \multicolumn{2}{|c|}{ Año 1999 } & \multicolumn{2}{c|}{ Año 2005 } \\
\hline Solo & $\mathbf{\%}$ & $\mathbf{n}$ & $\mathbf{\%}$ & $\mathbf{n}$ \\
\hline Madre & 18,50 & 37 & 30,00 & 60 \\
\hline Padre & 31,50 & 63 & 25,50 & 51 \\
\hline Pareja & 1,50 & 3 & 1,50 & 3 \\
\hline Amigo/amiga & 21,00 & 42 & 22,00 & 44 \\
\hline Pariente & 1,00 & 2 & 1,50 & 3 \\
\hline Otros & 19,50 & 39 & 13,00 & 26 \\
\hline NE & 4,00 & 8 & 3,50 & 7 \\
\hline \multicolumn{2}{|c|}{3,00} & 6 & 3,00 & 6 \\
\hline
\end{tabular}

Elaborado por: Myriam Trujillo

Fuente: Base de datos. Hoja de entrevista "Historia Clínica de la Adolescente - CLAP/OPS-OMS" 
También es importante analizar esta información desde otros saberes y factores, v.g., el nivel de ansiedad y angustia al que se enfrenta la adolescente ante la sospecha de un embarazo y los conflictos que esta situación podrían generar en la familia, como consecuencia de los cambios que la misma ha experimentado en las últimas décadas, le llevan a la búsqueda de respuestas de manera solitaria. Si bien, estadística y porcentualmente no es muy alta la incidencia de adolescentes que no conviven en relación de pareja respecto al año 1999, es clara la tendencia a incrementarse el número de adolescentes embarazadas que se ven forzadas a llevar "solas" su embarazo, sea por factores diversos que se intentarán comprender con los resultados y análisis posteriores.

Este fenómeno socio-urbano de la población adolescente, se explica desde el crecimiento demográfico, el cual se presenta elevado en la última década, en los sectores urbanos y especialmente en las ciudades de Quito y Guayaquil, en tanto que en sectores rurales es moderado y en algunos cantones es negativo. Según el Censo de 2001 la población urbana fue $61 \%$ y la rural 39\%, los hombres constituyen el 49,5\%, mientras que las mujeres son el 50,5\%. La población de 10 a 19 años proyectada fue del 20,89\% del total (UNFPA, 2006).

\section{Factores de riesgo personal y familiares}

El subcapítulo, pretende describir los factores de riesgo tanto biológicos, sociales y psicológicos que caracterizaron a la adolescente y su entorno familiar, y sirve como referencia para los análisis posteriores en este capítulo.

Aunque, de manera general los factores de riesgo médico se encontraban controlados, en ambos grupos de análisis tanto en el año 1999 como en el 2005, es interesante observar como otro tipo de factores más intrínsecos de la propia adolescente o de su entorno eran más recurrentes en el grupo del año 1999, así notamos que este grupo presentaba el doble de puntaje porcentual respecto a trastornos de carácter psicológico (14,5\% vs. 7,5\%), igual sucede con el uso de medicamentos o substancias no recetadas por el médico (28,5\% vs. $12,5 \%)$, o con los accidentes de intoxicación (14,0\% vs. 2,0\%). Es notoria esta precisión, que lleva a cuestionarse si este comportamiento no se encontraba influenciado por la presión social que sobre la problemática del embarazo precoz se tenía 
a fines del siglo pasado y el tabú sobre la sexualidad adolescente, y al mismo tiempo no encerraba un deseo no manifiesto verbalmente de interrumpir prematuramente el embarazo.

El instrumento que se utiliza en la unidad de consulta externa del Proyecto HGOIA-AECI, recoge importante información sobre factores biológicos de antecedentes familiares de la adolescente embarazada. En este sentido, se considera factor de riesgo predominante entre la población adolescente de 1999 la procedencia de madres adolescentes ( $47 \%$ vs. $28 \%$ en el 2005 ), mientras que entre las adolescentes en el 2005, se observa un incremento considerable del consumo de alcohol o drogas entre los miembros de su familia, incremento de 7,5 puntos en comparación al grupo de 1999 ( $5 \%$ vs. $12,5 \%$ ).

\section{Familia: entorno y características}

Información relevante en esta sección tiene que ver con el entorno familiar inmediato en que se desenvuelve la adolescente embarazada. Se comprobó que para 1999 un 53\% de ellas vivían con su madre y un 39\% con sus padres, más para el 2005 este porcentaje ha disminuido en $12,5 \%$ y un $13 \%$ respectivamente, lo que nos devela una cierta fragilidad en la conformación de la familia en la sociedad moderna. En tanto las adolescentes que conviven con una pareja en el período de embarazo no han variado significativamente.

El nivel de escolaridad de los padres de las adolescentes embarazadas, no parece ser un factor determinante entre los riesgos adolescentes aunque se evidencia un porcentaje de escolaridad primaria y secundaria baja tanto entre madre y padre de adolescentes con embarazo precoz, estos datos porcentuales aparentemente se manifiestan similares con muy poca variación entre los dos años de estudio.

La estabilidad de ingresos económicos según el tipo de trabajo que posee el padre y/o la madre, reporta que en su gran mayoría las adolescentes con embarazo precoz provienen de familias con trabajos estables, aunque, las madres tienen un alto porcentaje de desocupación e inestabilidad laboral, en relación al padre que mantiene un estado de relación laboral más estable económicamente.

Aunque el formato de la encuesta que realiza el proyecto en los años en cuestión no nos da muchos criterios de interpretación en cuanto a la percepción que 
posee la familia respecto a la situación de embarazo precoz en su hija adolescente, por cuanto los criterios son muy vagos al referirse a percepción buena, regular o mala. En todo caso, se observa que ha existido un cambio bastante significativo en la percepción que las familias poseen del embarazo adolescente, así respecto al evento para 1999, el 74,5\% las percibían como "regular", mientras que para el año 2005 esta percepción ha variado a positiva "buena" para el 70,5\% de las familias. Es decir, se ha percibido un cambio de comportamiento de la familia y por ende de la sociedad en su manera de percibir el embarazo adolescente.

\section{Vivienda, educación, trabajo, vida social y hábitos}

Es necesario conocer las condiciones sociales en las que se presenta el embarazo precoz entre las adolescente, por ello, por un lado se analizó información que permita comparar si la falta de servicios básicos y de oportunidades eran desencadenantes de embarazos precoces. Con respecto a su vivienda y las condiciones en las que estas se encontraban, en los dos años analizados afirmaron en un $100 \%$ contar con servicios de energía eléctrica, y solo un $95,5 \%$ para 1999 aseguraron tener agua potable al interior de su vivienda, lo cual entre la población del 2005 ha disminuido al $90 \%$.

Para analizar el perfil educativo de la adolescente embarazada, se investigó información relevante, que nos indica si estudia o no, nivel de escolaridad, si tenía o no problemas en el establecimiento educativo, así como deserción y posibles causas de la misma.

Comprobamos a partir del análisis de la información, que en el período de investigación correspondiente a 1999 el 75,5\% de las adolescentes manifestaban no estudiar para el momento que inician sus chequeos médicos de su embarazo. En tanto, para el año 2005 este porcentaje ha disminuido notablemente, lo que lleva a pensar que las adolescentes del último periodo de investigación efectivamente se mantienen en el sistema escolar a pesar de su embarazo precoz, y que la familia y la sociedad de alguna manera ha cambiado su percepción respecto a la problemática de embarazo adolescente, teniendo en cuenta que en los últimos años ha existido un movimiento social muy fuerte en cuanto al respeto del derecho a la educación, sin discriminación en su estado de embarazo. 
El nivel de instrucción de la adolescente no ha variado significativamente en ambos grupos poblacionales, tanto en 1999 como en el 2005, las adolescentes con embarazo precoz se encontraban en porcentajes altos $76 \%$ y $79,5 \%$ respectivamente, cursando la instrucción secundaria.

De la población que afirmó tener instrucción secundaria en 1999 el 54\%, sólo había llegado a cursar entre el segundo y tercer año de secundaria, es decir, lo que el sistema educativo cataloga como el noveno y décimo año de educación básica, en tanto para el año 2005 el 48,4\% afirmaba encontrarse cursando la instrucción del bachillerato. En los dos años estudiados 1999-2005, las adolescentes manifestaron no presentar problemas de importancia en la escuela o colegio, a pesar que en el año 2005 se presenta un alto porcentaje de adolescentes que no responden a esta pregunta.

Como consecuencia del embarazo para 1999 se registra un alto porcentaje de deserción escolar por parte de las adolescentes $64,5 \%$, para el 2005 como se había manifestado en párrafos precedentes la tendencia de desertar del sistema educativo a descendido, es tal que para este año la deserción escolar en las adolescentes solo llegaba a un $45 \%$, aunque indudablemente el porcentaje aun se mantiene alto, es rescatable que en un período de casi seis años haya descendido casi veinte puntos porcentuales.

Distribución porcentual según deserción escolar

\begin{tabular}{|l|c|c|c|c|}
\hline \multirow{2}{*}{ DISTRIBUCION } & \multicolumn{2}{|c|}{ Año 1999 } & \multicolumn{2}{c|}{ Año 2005 } \\
\hline & \% & N & \% & n \\
\hline $\mathrm{Si}$ & 64,5 & 129 & 45,0 & 90 \\
\hline $\mathrm{No}$ & 32,0 & 64 & 46,5 & 93 \\
\hline $\mathrm{Ne}$ & 3,5 & 7 & 8,5 & 17 \\
\hline TOTAL & 100 & 200 & 100 & 200 \\
\hline
\end{tabular}

Elaborado por: Myriam Trujillo

Fuente: Base de datos. Hoja de entrevista "Historia Clínica de la Adolescente - CLAP/OPS-OMS" 
$\mathrm{Al}$ analizar, la causa de esa deserción del sistema escolar, las adolescentes coinciden, en ambas muestras, que el embarazo es la primera causa $(42,5 \% \mathrm{y}$ $31,1 \%$ ) y que la situación económica, es la segunda causa $(21,7 \%$ y $19 \%)$, entre ambas causales se llega a un promedio de $64,3 \%$ en el año 1999 y $50,1 \%$ en el año 2005.

Si existe un alto porcentaje de adolescentes que abandonan el sistema escolar, se hace necesario conocer, a qué otras actividades se dedican durante el período de su embarazo. Se pudo encontrar que en 1999 el 17\% trabajaba durante el período de su embarazo, en tanto que para el 2005 este promedio disminuyó a un $14 \%$ de ellas. Hay que considerar que en ese mismo año el $39 \%$ de las adolescentes manifestó que había trabajado, pero ahora se encuentra desempleada.

Entre las adolescentes embarazadas que afirmaron que trabajan, la edad promedio de inicio de trabajo para 1999 fue 13,9 años; en cambio para el año 2005, la edad promedio bajó a 12,4 años. Lo que lleva a reflexionar sobre las diversas aristas que tiene presente esta problemática. El tiempo de dedicación al trabajo por parte de las adolescentes es en su mayoría de jornada completa, según lo manifiestan 82,4\% en el año 1999 y 71,4\% en el 2005.

En 1999, el 82,3\% las adolescentes responden que trabajan por necesidad económica, el $14,7 \%$ lo hace porque le gusta y un $2,94 \%$ por buscar independencia; en el año 2005 , el $50 \%$ lo hace por necesidad monetaria, el $28,5 \%$ porque le gusta, y hay un incremento importante, $17,8 \%$, que lo hace con miras a encontrar autonomía e independencia.

La mayoría de trabajos de las adolescentes $76,8 \%$ en 1999 no están legalizados, es decir, no contaban con algún tipo de contrato, ni afiliación al seguro; en 2005 el 50\% manifestó contar con un trabajo legalizado, que aunque no contaban con seguro mantenían algún documento contractual entre empleador y adolescente. Al indagar sobre el tipo de trabajo que realiza la adolescente, la mayoría de ellas trabaja como empleada doméstica, 35,3\% o como vendedora dependiente $17,7 \%$ en 1999 ; y $21,4 \%$ en el año 2005 , seguido por trabajos informales como vendedora dependiente o comerciante.

Para caracterizar el perfil socio familiar de la adolescente embarazada, es determinante conocer las prácticas de vida social que lleva la adolescente, para 
ello del instrumento "hoja de entrevista de la Historia Clínica", se ha analizado los siguientes criterios:

1. Aceptación social: según la percepción de las adolescentes embarazadas, tanto en su ambiente familiar como social son aceptadas en su mayoría, así en 1999 el 92,5\% y para el año 2005 en un 89,5\%.

2. Aceptación del novio: similar porcentaje de aceptación indican, al consultárseles si después de conocer su estado de embarazo siguen siendo aceptadas por sus novios, 82\%, 78,5\% en 1999 y 2005 respectivamente. Sólo un porcentaje bajo en lo global pero duplicado si comparamos ambos años de estudio no acepta a su pareja en estado de embarazo, hallando este porcentaje en 7,5\% en 1999 y duplicado en $14 \%$ para el año 2005.

3. Aceptación por parte de su grupo de amigos-as: al parecer, respecto a la aceptación de su embarazo por parte de su núcleo de amigos las adolescentes en 1999, se negaron a contestar en porcentajes altos 70,5\%; en relación al año 2005 en el que las adolescentes manifestaron que su estado de embarazo era aceptado por sus amigos, $79 \%$.

4. Otras actividades: concurren otras actividades que realizan las adolescentes, pero la que más identifican ellas como actividad determinante es "quehaceres domésticos", tanto en 1999 y 2005, 90,8\% y 76\% respectivamente. Esto evidencia, en gran medida, su aislamiento de sus grupos sociales y por ende de las actividades grupales.

Respecto a la vida social durante el embarazo adolescente es importante puntualizar y contraponer los diversos análisis de cada sub-ítem, por ejemplo:

Se aprecia una disminución de la percepción que tiene la adolescente respecto a la aceptación de la familia a su embarazo, aunque esta se mantenga elevada de manera general, se presenta discursiva y declarativa, "la familia tenderá a manifestar su indiferencia o rechazo a través de múltiples actitudes, llenándola de mayores responsabilidades en el hogar o generando un clima recriminatorio" (Rodríguez, Miguel, 2005). En un 57,5\% las adolescentes disminuyen sus actividades de carácter grupal y aumentan en tanto incrementan sus 
labores domesticas, el 90,8\% en 1999 y el 76\% en el 2005 manifiestan dedicarse a "quehaceres domésticos" durante su embarazo. Para estudios posteriores es recomendable el análisis de factores de aceptación familiar y sus actitudes ante el embarazo adolescente, las labores de la adolescente y sus repercusiones anteriores o posteriores a la deserción escolar.

La percepción de aceptación del grupo de amigos es alta en el 2005, pero al mismo la actividad grupal no supera el $20 \%$ lo que podría percibirse como una falsa aceptación por parte de los amigos de la adolescente embarazada o por la influencia que el grupo familiar tiene realmente sobre esta, como se explicó en el párrafo anterior, siendo la familia la que limita las actividades grupales de la adolescente, a fin de responsabilizarle mayormente de su nueva condición de "gestante", "madre".

\section{Sexualidad y situación psicoemocional}

a. Sexualidad: en la encuesta se recogió información detallada sobre el perfil del comportamiento sexual de las adolescentes, a partir de los 12 años aproximadamente presentaron su primera menstruación, en un 37,5\% y un $33,5 \%$ en los años 1999 y 2005 respectivamente, para ambos años estudiados, no ha existido mayor cambio en el promedio de edad de la menarquia, en ambos el promedio es de 12,6 años y 12,5 aproximadamente.

En cuanto a saber si ellas consideraban necesario que se les brinde mayor información sobre sexualidad. En 1999, el 91,5\% manifestó que requerían mayor información sobre temáticas de sexualidad, en tanto para el 2005 este porcentaje ha disminuido al $64 \%$, esto lleva a colegir que las adolescentes embarazadas de esta última muestra consideran poseer la información necesaria sobre sexualidad, o en mayor proporción reconocen no necesitar de tal información.

Hemos generado a través de los medios de comunicación un erotismo de consumo, que ha dirigido en forma preferente sus esfuerzos mercantilistas hacia los jóvenes. Estudios demuestran que la TV. y otros medios se han convertido en los principales educadores sexuales. La adolescente actual, si bien, tiene acceso a mayor información externa en el área de la sexualidad, mientras que la 
educación formal del colegio e informal de la familia sigue siendo insuficiente (analfabetismo sexual) (Naranjo, 2000).

Un factor de riesgo importante, para el análisis del perfil socio familiar de la adolescente, tiene que ver no solo con el inicio temprano de relaciones sexuales, sino con el comportamiento promiscuo en las mismas. En tal virtud la encuesta contemplaba un análisis sobre el número de parejas con las que ha mantenido relaciones sexuales. Es interesante en este punto del análisis que a diferencia de cualquier pronóstico para el 2005 solo un 3\% de las adolescentes manifestó haber tenido varios compañeros sexuales, a diferencia del 7\% y $8 \%$ del año 1999, que puede deberse, como lo vimos anteriormente al alto índice de desconocimiento de la sexualidad y sus implicaciones que manifestó este grupo poblacional.

El poco conocimiento del uso de anticonceptivos y la inadecuada información por parte de entorno familiar, escolar y social, hacen que las adolescentes mantengan relaciones sexuales sin la utilización de métodos anticonceptivos, afirmando que nunca utilizan un método de anticoncepción en porcentajes elevados.

Otro factor importante, analizado entre las adolescentes encuestadas, fue preguntarles si habían padecido algún tipo de enfermedades de transmisión sexual (ETS), a lo cual la mayoría manifestó no haber contraído ningún tipo de ETS en 1999 al igual que en el 2005. Solo un porcentaje mínimo 1,5\%, como se aprecia en la tabla siguiente, padeció en el 2005 de alguna enfermedad ETS. Esto no necesariamente es señal de un incremento de la promiscuidad sexual de las adolescentes de esta muestra en consideración a la muestra de 1999.

A la pregunta ¿Ha tenido algún otro embarazo?, la mayoría de las adolescentes manifiestan no tener ningún embarazo anterior, en un $83 \%$ para 1999 , al igual que el 78,5\% en el año 2005. A diferencia de la muestra de 1999, las adolescentes del grupo del año 2005 habían manifestado en un $21 \%$ que efectivamente habían tenido anteriormente otro embarazo. En promedio, el inicio de relaciones sexuales en adolescentes no ha variado significativamente en los últimos cinco años, en 1999 fue de 15,3 años y en al año 2005 varia a 15,6 años.

El abuso sexual de adolescentes se analizó como factor y antecedente de importancia respecto al embarazo precoz de adolescente, por ello, también se 
les indagó si su primer contacto sexual fue voluntario o por abuso sexual, la mayoría de entrevistadas afirma que no hubo abuso y que sus relaciones sexuales fueron voluntarias. Tan solo un $6 \%$ en 1999 y un 4,5\% en el año 2005, recaban que hubo abuso sexual por parte de algún familiar (primos, cuñados, tíos).

b. Situación Psicosocial y proyectos de vida: La encuesta recogió información detallada sobre varios aspectos que tienen que ver con la situación Psicosocial que atraviesa la adolescente embarazada, encontrándose los siguientes resultados. Las adolescentes embarazadas, manifestaron sentirse conformes con su imagen corporal; y respecto a su auto percepción respecto a su situación actual, un 49,5\% se sienten alegres en 1999 y $51,5 \%$ para el año 2005 , tristes $6 \%$ y 25,5 respectivamente, nerviosas o con carácter variable en 1999 fue el 20,5\% y en el año 2005 un 9,5\%; muy tímidas un 16,5\% y 7\% en los años 1999 y 2005.

Es interesante reconocer que después de la valoración que las adolescentes les dan a su grupo familiar cercano en porcentajes muy similares responden que la experiencia del embarazo, les ha dado mayor claridad para la construcción de un proyecto de vida, tanto para el $29,5 \%$ en 1999 y el $23,5 \%$ en el 2005 , la situación de embarazo precoz es confuso en la definición de su proyecto personal de vida.

\section{Perfil del embarazo adolescente}

En función de este tema se elaboró y aplicó un instrumento tipo encuesta a cincuenta adolescentes, para identificar y caracterizar el embarazo adolescente como una problemática social que afecta a la sociedad moderna y muy particularmente la nuestra. Este capítulo se sustenta en la encuesta mencionada, que se encuentra como anexo a la presente investigación, y fue aplicada a cincuenta adolescentes usuarias del proyecto HGOIA-AECI del Hospital Gíneco-Obstétrico de Quito, esta vez, se recolecta información de quienes asistían, tanto para control prenatal como de puerperio post parto, a la consulta externa del mismo, durante los meses enero y febrero del año presente. El universo de estas encuesta fueron "adolescentes menores" en un porcentaje del $12 \%$ y el $88 \%$ "adolescentes mayores", que en términos médicos se encuentran comprendiendo el rango de diez a catorce años y quince a diecinueve años respectivamente. 
El embarazo adolescente es una situación que se da, según la información obtenida del grupo de estudio, en adolescentes que mantienen una sexualidad activa y conviven como pareja, $44 \%$, en tanto que el $38 \%$ aún depende de sus padres y conviven con ellos.

En cuanto a escolaridad y deserción, el embarazo adolescente tiende en el $54,55 \%$ de las adolescentes a provocar su deserción escolar, ya no estudian, en un porcentaje mínimo 3,03\%, son los padres que presionan a la adolescente embarazada a fin de que abandone la escuela y se dedique a "ayudar en la casa". Volviendo a las dos categorías médicas, sobre adolescentes menores y mayores, se hizo el análisis, cabe resaltar que según los resultados la mayor parte de las adolescentes de diez a catorce años suspenden sus estudios por este motivo, así el $83 \%$ de las adolescentes, y un $64 \%$ entre las edades de quince a diecinueve años.

La escolaridad en embarazos adolescentes, varía en ambos grupos, en el grupo de diez a catorce años tiene primaria completa en un 50\%, en tanto las de quince a diecinueve años solo en un $25 \%$ terminan la primaria, el 16,7\% del grupo de las adolescentes menores y un $2,3 \%$ de las adolescentes mayores no llegaron a terminar la primaria, el $33 \%$ de adolescentes menores versus un $63,6 \%$ de adolescentes mayores están cursando la secundaria y del grupo de quince a diecinueve años el 9\% terminó la educación secundaria. La mayor parte, el $60 \%$ de las adolescentes tiene secundaria incompleta, dato que podría afirmar el motivo de deserción "el embarazo precoz", un 28\% tiene primaria completa, un $4 \%$ primaria incompleta y un $8 \%$ terminó su bachillerato.

La actividad económica y no sólo la educativa, se ven afectadas por el embarazo adolescente, de los resultados obtenidos el $86 \%$ de la población encuestada manifiesta no trabajar y la mayoría afirma que no lo hace por su situación de embarazo. En el análisis por grupo develamos que el 100\% de las adolescentes menores no trabajan, en cuanto al grupo de adolescentes entre quince a diecinueve años el $16 \%$ si trabajan versus un $82 \%$ de adolescentes que no mantiene ninguna actividad laboral. El promedio de edad, para el inicio de las relaciones sexuales entre las adolescentes embarazadas es de 15,06 años, el $26 \%$ de ellas inicia su vida sexual a los catorce años.

Es interesante la información brindada por las adolescentes, al preguntárseles ¿En qué circunstancia y lugar tuvo su primera relación sexual? El 78\% 
manifestó haberlas iniciado en su casa y el $84 \%$ afirma que su relación sexual fue con su consentimiento, el 14\% considera que se vio forzada (para retener la pareja o por la prueba de amor) y un $2 \%$ manifestó que se produjo con violencia o abuso.

Es importante señalar que las "niñas" adolescentes que inician sus relaciones sexuales en un $60 \%$ tienen un rango de edad de entre doce y quince años; en un $80 \%$ tales relaciones se inician en sus hogares, a lo cual habría que añadirle el $16 \%$ de las cuales son forzadas o violentas. Menor grado porcentual al inicio de relaciones sexuales adolescentes en un $22 \%$ se dan en ambientes externos o fuera de casa ( $6 \%$ en casa de amiga o pareja; el $6 \%$ en un paseo; $2 \%$ casa de tía u hostal).

E1 70\% de las adolescentes manifestó haber mantenido su primera relación sexual con su enamorado, tal vez por presión de su pareja pidiendo su prueba de amor o primera ilusión, el $24 \%$ lo hace con su novio, y un $6 \%$ con algún amigo. Aunque no se ha definido claramente estas categorías entre las adolescentes a su decir el novio es con quien se tiene la promesa de matrimonio y se considera una relación más estable y de mayor compromiso que la de enamorado.

Otra característica importante del embarazo adolescente tiene que ver con la información que ellas poseen sobre sexualidad y riesgos, pero al cuestionárselas sobre el uso de métodos anticonceptivos, el $78 \%$ de ellas respondió que no los habían utilizado nunca, y al preguntarles sobre la causa de no consumirlas, el 53,85\% no especificó el motivo y el 30,7\% respondió que no sabía que podía evitar el embarazo.

Entre el grupo de adolescentes menores, $100 \%$ manifestaron no utilizar ningún método anticonceptivo al momento de mantener relaciones sexuales, en tanto que el grupo adolescentes mayores solo el $9 \%$ manifestaron que si utilizaron algún tipo de anticonceptivo previo a la relación sexual. 


\section{Distribución porcentual según uso de anticonceptivo}

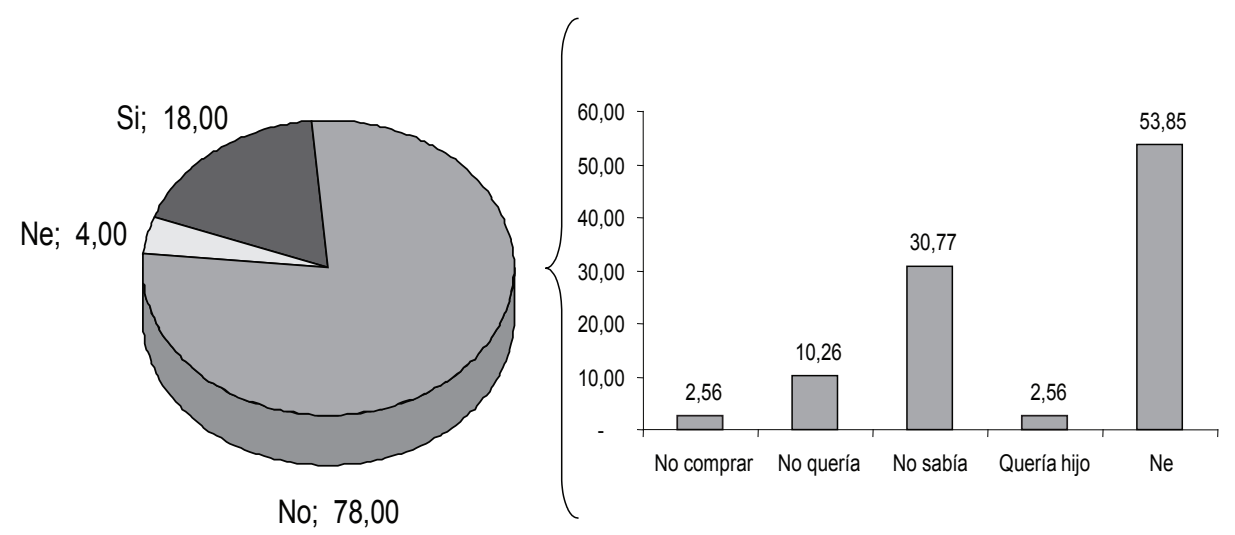

Elaborado por: Myriam Trujillo

Fuente: Encuesta a cincuenta adolescentes. Consulta Externa de Adolescencia HGOIA

El $82 \%$ de adolescentes se embarazaron luego de varios contactos sexuales, en tanto que el $18 \%$ afirmó que se habían embarazado con su primera experiencia sexual. Es importante reconocer que el embarazo adolescente no se da previo a la convivencia con la pareja, el $78 \%$ de las adolescentes manifestó que no convivía aún con su pareja.

Por los resultados tampoco se podría afirmar o pensar que el embarazo adolescente promueve la convivencia en pareja de las adolescentes, por cuanto a preguntárseles si después del embarazo, es decir en la actualidad, convivían como pareja, el 62\% manifestó que no, por diversas causas como:

- "no sé dónde está"; "no hemos decidido"; "problemas familiares"

• "él no quiere"; "aún no nos casamos"; "vive lejos"; "no es necesario

- "mis padres no lo quieren"; "no quería hacerse responsable"; "se marchó"; "no puedo obligar a nadie que esté conmigo"

En comparación con los resultados de la tabla anterior, sólo el 20\% del total de adolescentes que dijeron que no convivían en pareja al embarazarse, lograron iniciar la vida en pareja después del embarazo. Verificándose en los resultados una notoria ausencia del padre en la vida del futuro neonato, sean estos 
por factores de abandono "no sé donde está" "vive lejos" o "no quiere hacerse responsable; o de exclusión "mis padres no lo quieren", "no es necesario".

El 64\% de los embarazos adolescentes son asumidos con "responsabilidad" por parte de los varones "causantes" de los mismos, es decir, por los padres asumen la paternidad y reconocen como suyos al producto del embarazo adolescente, del total de embarazadas el 36\% manifestó que los "causantes" del embarazo niegan la paternidad.

Al investigar si el embarazo era intencional, las adolescentes manifestaron que no habían pensado en la posibilidad de embarazarse al momento que mantener relaciones sexuales, el 74\% tenía desconocimiento de las consecuencias a futuro, el $26 \%$ por el contrario manifestaron que sabían que corrían el riesgo de embarazarse, y si pensaban en esa posibilidad. Pero en ningún caso habría habido la intención de embarazarse.

La pregunta subsiguiente era conocer si, a pesar de la posibilidad de un embarazo, la adolescente efectivamente quería embarazarse, a esta pregunta respondieron efectivamente el $22 \%$ de ellas. Se pudo observar que si bien el $74 \%$ de los embarazos adolescentes no son planificados ni pensados por ellas, respondieron que no pensaron embarazarse; el 80\% de ellas asumen optimistamente su embarazo y dicen que si están contentas con ello. Los motivos más relevantes manifestados son los siguientes: "ahora le voy a cuidar"; "es lo único que tengo en la vida"; "es algo mío"; "orgullosa de sentirme mamá"; "tengo a mi bebé".

Efectivamente el embarazo adolescente colma algunas carencias adolescentes como se ve en las expresiones arriba mencionadas "ahora le voy a cuidar", que recoge carencias de afectividad; "es lo único que tengo en la vida", "es algo mío", "es mi hijo", carencias de posesión y propiedades; "orgullosa de sentirme mamá" que recoge carencias de autoestima, etc. Esto parece manifestar que el hijo colma positivamente muchas carencias y necesidades de la adolescente, como si con el hijo la vida de la adolescente fuera menos precaria.

La potencialidad de la condición de "madre" en el embarazo adolescente, satisface al 86\% de las adolescentes, en tanto el 14\% de ellas afirma no acepta a su embarazo y la potencial condición de madre, a esto ellas declaran que "no fue por amor", "mucha responsabilidad", "no lo esperaba", etcétera. 
El embarazo adolescente según manifestaron las adolescentes en 98\% afirman que el embarazo les cambió totalmente su proyecto de vida, por cuanto exteriorizan los motivos siguientes: "me da más responsabilidad"; "debo mantener a mi hijo"; "más responsable, voy a trabajar"; "mi bebé va a necesitar"; "no puedo seguir estudiando"; "tengo que buscar trabajo"; "responsable y estudio para superarme". El temor a la responsabilidad es el punto más relevante durante el embarazo adolescente.

\section{Conclusiones}

Se puede establecer, como conclusión de éste capítulo, a partir de la entrevista in situ con cincuenta adolescentes, la magnitud de la problemática social del embarazo precoz, los criterios e información brindada por las propias "afectadas", afirman la complejidad del mismo como problema sociológico.

Concretamente, el embarazo adolescente, se caracteriza por darse entre adolescentes que tienen una vida sexual activa y comparten con una pareja o compañero sexual. Tiende a promover la deserción escolar y el trabajo adolescente. Por otro, el inicio de la actividad sexual tiende a iniciarse en los propios hogares de las adolescentes, y por voluntad propia, a diferencia del criterio del sentido común que tiende a explicar este como un fenómeno que se da fuera del ambiente familiar y por actos de fuerza o engaño. Esto también conlleva a reflexionar sobre el papel trascendente que juega la familia en el incremento de esta problemática.

En lo que respeta al "deseo" de la adolescente de embarazarse por retener o convivir de manera permanente con su "novio", es interesante comprobar que un porcentaje mínimo lo manifestaba, siendo el mismo porcentaje que convivía previo a su embarazo con una pareja. Aunque existe un incremento en el número de adolescentes que inician vida de pareja después del embarazo, no se podría considerar significativo, dado que $62 \%$ de las adolescentes se mantendrán solas durante y después del embarazo.

Se hace importante relevar aspectos como el incremento de la "autoestima" en la adolescente embarazada, para futuras observaciones, si bien inicialmente se destaca en el perfil social familiar del embarazo adolescente factores de so- 
ledad, angustia y temor, se podría considerar también factores de poca comunicación en el interior de su núcleo familiar, por lo mencionado en el capitulo anterior los "cambios por los que atraviesa la familia". Se observa factores como "la afectividad", descargar afecto al ser por nacer le ayudan a valorarse como sujeto "amante", con y en capacidad de amar y entregarse, otros como "la posesión" el valor de tener "algo" que ciertamente considera totalmente suyo, ayudan de cierto modo a incrementar su propia valoración.

\section{Bibliografía}

\section{BOLETINES INFORMATIVOS ESPECIALES}

http://www.hoy.com,ec/noticianue.asp?row=215,102:fecha:17/02/2006 noticias del ecuador y del mundo hoy online.

CEPAR

2005 Ecuador Endemian 2004 Informe final-Encuesta demográfica y de salud materna e infantil. Quito.

CEPAR, Et al.

2005 Encuesta Demográfica y de Salud Materno e Infantil. Quito.

CICLO MENSTRUAL

CLADEM

http://es.wikipedia.org/wiki/ciclo_menstrual

2004 Derechos Sexuales y Reproductivos en el Ecuador. CLADEM-Diagnóstico Nacional.

\section{CLADEM}

2004 Derechos Sexuales, Derechos Reproductivos en el Ecuador.

CONAMU

2004 Ecuador 10 años después: Análisis del Cumplimiento de los Compromisos del Ecuador en la Conferencia Internacional sobre Población y Desarrollo el Cairo 1994. Consejo Nacional de las Mujeres. Quito.

CONASA

2004 Consulta preparatoria por el Comité Nacional de salud y Derechos sexuales y Reproductivos.

CONASA

2002 Marco General de la Reforma Estructural de la salud en el Ecuador. Ministerio de Salud Pública. Quito. 
CONASA

2006 Politica Nacional de Salud y derechos sexuales y reproductivos. 1era. Edición. Quito.

COLL, Ana. et al.

2001 Atención a la Adolescente Embarazada. Universidad Centroccidental Lisandro Alvarado.

DURKHEIM Emile http://es.wikipedia,org/wiki/Emile_Durkheim

EMBARAZO ADOLESCENTE

http:/www.monografías.com/trabajos32/embarazo-adolescente/embarazoadolescente.

ENDRIGTERAN, Neke

2000 Maternidad pobreza en el Ecuador. Quito.

FAMILIA Y EDUCACIÓN

http://www.sociologicus.com/portemas/familia/familiayeducacion.html

FREÍD, S.

1992 Sobre la sexualidad femenina. Tomo XX. Amorrortu Editores. Buenos Aires.

GÓMEZ, Virginia. et al.

2001 Violencia Intrafamiliar y de Género. Torrescal. Quito.

GUTIÉRREZ, Enrique

1998 Atención a la Adolescente Embarazada. Universidad Centroccidental Lisandro Alvarado.

GUTIÉRREZ Marisol

El Embarazo Adolescente http://www.monografías.com/trabajos16/embarazo-en-adolescentes/ embarazo-en-adolescentes.zip. amarisola@hotmail.com

HAKKERT, Ralph

2003 Fecundidad deseada y no deseada en América Latina. UNFPA CST LAC.

INFORME MORTALIDAD INFANTIL Y MATERNA

http://www.cor.opsoms.org/TextoCompleto/documentos/Informe_Mortalidad_infantil_y_materna.pdf

INFORMES INFANCIA

http://www.periodismosocial.org.ar/area._infancia._informes.efm?ah=101

ADDALENO, Matilde. et al.

1995 La Salud del Adolescente y del Joven. OPS-ONS. Washington, D.C. MSP.

1995 Manual de Normas y Procedimientos para la Atención Integral a la Salud de los/las Adolescentes con énfasis en la Salud Reproductiva. Quito. 
2005 Política Nacional de Salud, Derechos Sexuales y Reproductivos. Quito.

NARANJO, Jorge

2000a Diagnóstico Situacional y el Marco Conceptual para la Atención Integral de Adolescentes y Jóvenes en el Ecuador. Documento técnico $\mathrm{N}^{\circ} .1$ Quito.

2000b El Perfil de las Usuarias Adolescentes. Proyecto HGOIA-AECI, 12. 1999 a 2000. Ecuador.

ORGANIZACIÓN MUNDIAL DE LA SALUD

1977 Necesidades de Salud de los Adolescentes. Informe del Comité de Expertos Serie de Informes Técnicos. Ginebra.

RODRÍGUEZ, Miguel

2005 Aceptación del embarazo adolescente, por el grupo familiar: barrio 12 de Octubre-Barquisimeto. Universidad Centro Occidental "Lisandro Infantiles y Alvarado": Barquisimeto.

SÁNCHEZ Parga, José

2004a Orfandades Infantiles y Adolescentes: Introducción a una sociología de la infancia. Abya Yala. Quito.

Poligrafiados y apuntes en clases.

2004b Revista Universitas. Editorial Don Bosco. Cuenca.

SCHWARCZ, Sala

1998 Obstetricia. el Ateneo. Argentina.

UNICEF

2003 Observatorio Ciudadano de los Derechos de la Niñez y Adolescencia - Estado de la Niñez y Adolescencia en el Ecuador. Quito.

2004 Observatorio Ciudadano de los Derechos de la Niñez y Adolescencia - Estado de la Niñez y Adolescencia en el Ecuador-Sexo y Reproducción en los Adolescentes. Quito.

UNIDAD DE PROGRAMAS SANTA ELENA

2004 Conozcamos Nuestros Derechos. MSP.

UPS

2002 Revista Universitas año I- No 2. Familia. Editorial Don Bosco. Cuenca.

WOODWARD, Kathy: Et Al,

1995 La salud del adolescente y del joven. Organización Panamericana de la Salud. OPS. 